\title{
Triton binding energy with realistic statistical uncertainties
}

\author{
R. Navarro Pérez, ${ }^{1, *}$ E. Garrido, ${ }^{2, \dagger}$ J. E. Amaro, ${ }^{1, \dagger}$ and E. Ruiz Arriola ${ }^{1, \S}$ \\ ${ }^{1}$ Departamento de Física Atómica, Molecular y Nuclear and Instituto Carlos I de Física Teórica y Computacional Universidad de Granada, \\ E-18071 Granada, Spain \\ ${ }^{2}$ Instituto de Estructura de la Materia, CSIC, Madrid, Spain
}

(Received 30 July 2014; revised manuscript received 3 September 2014; published 6 October 2014)

\begin{abstract}
We compute the binding energy of triton with realistic statistical errors stemming from $N N$ scattering data uncertainties and the deuteron and obtain $E_{t}=-7.638(15) \mathrm{MeV}$. Setting the numerical precision as $\Delta E_{t}^{\text {num }} \lesssim$ $1 \mathrm{keV}$ we obtain the statistical error $\Delta E_{t}^{\text {stat }}=15(1) \mathrm{keV}$ which is mainly determined by the channels involving relative $S$ waves. This figure reflects the uncertainty of the input $N N$ data, more than two orders of magnitude larger than the experimental precision $\Delta E_{t}^{\exp }=0.1 \mathrm{keV}$, and sets a limit on the realistic precision that can be reached. This suggests an important reduction in the numerical precision and hence in the computational effort.
\end{abstract}

DOI: 10.1103/PhysRevC.90.047001

PACS number(s): 21.10.Dr, 13.75.Cs, 21.30.Fe, 21.45.-v

One of the main challenging goals in theoretical nuclear physics is the ab initio determination of binding energies of atomic nuclei. The accepted protocol consists of undertaking a quantum multinucleon calculation from the knowledge of few-body forces. The simplest case were such a program has been most often investigated is the binding energy of the triton, a stable system consisting of two neutrons and a proton with an experimental mass defect given currently by $M_{t}-2 m_{n}-$ $m_{p}=E_{t}^{\exp }=-B_{t}^{\exp }=-8.4820(1) \mathrm{MeV}$. Already in the mid1930s quantum mechanical theoretical studies of triton binding allowed researchers to establish essential properties of the nuclear force: its finite range as well as the existence of neutron-neutron interactions (see, e.g., Refs. [1,2] for early reviews). The increasing precision in our knowledge of the two-body interaction has strongly motivated the developments in solving the computationally expensive $3 N$ problem (see, e.g., [3-6]). While this was partly aimed at establishing the need for $3 N$ forces, high numerical precision in conjunction with realistic and precise nucleon-nucleon interactions has become a major issue by itself in few-body computational methods. In Refs. [7-9] benchmarking precisions of $\Delta E_{t}^{\text {num }}=$ $10,0.1,0.01 \mathrm{keV}$, respectively, have been achieved within different schemes.

However, nucleon-nucleon potentials determined from data inherit statistical fluctuations that propagate to the triton theoretical energy into a genuine statistical error $\Delta E_{t}^{\text {stat }}$. A pioneering and forgotten attempt already looked at the consequences for triton binding based on an analysis of the inverse scattering in the ${ }^{1} S_{0}$ channel [10]. In the present paper we quantify for the first time the uncertainty of triton energy $\Delta E_{t}^{\text {stat }}$ stemming from a complete statistical analysis of 6713 selected nucleon-nucleon scattering data. In our analysis we consider a particular $N N$ potential and disregard the role of $3 N$ forces. While the particular representation of the $N N$ potential may induce changes in the triton binding energy of at most

\footnotetext{
*rnavarrop@ugr.es

†e.garrido@csic.es

†amaro@ugr.es

§earriola@ugr.es
}

$5 \%$, we do not expect significantly larger fluctuations for the statistical error estimated here. Our findings below confirm this naive expectation.

The main and most reliable sources of information for the $N N$ interaction are the deuteron energy and the more than $8000 n p$ and $p p$ scattering data below pion production threshold published during the last 65 years. These will be denoted as $O_{i}^{\exp } \pm \Delta O_{i}$, with $i=1, \ldots, N$ and will be regarded as normally distributed variables. In the classical statistical approaches one proposes a given $N N$ interaction $V_{N N}(\mathbf{p})$ depending on a set of parameters $\mathbf{p}=\left(p_{1}, \ldots, p_{P}\right)$ which, by solving the two-body Schrödinger equation, generates a set of scattering observables $O_{i}(\mathbf{p})$ with $i=1, \ldots, N$. The parameters are determined by a least-squares $\chi^{2}$ fit,

$$
\min _{\mathbf{p}} \chi^{2}(\mathbf{p})=\min _{\mathbf{p}} \sum_{i=1}^{N}\left(\frac{O_{i}^{\exp }-O_{i}(\mathbf{p})}{\Delta O_{i}^{\exp }}\right)^{2} \equiv \chi^{2}\left(\mathbf{p}_{0}\right) .
$$

A high quality potential is one verifying $\chi^{2} / v \sim 1$, with $v=$ $N-P$. Since the Nijmegen group analysis in 1993 [11] a set of high quality potentials have emerged fitting their contemporary databases [12-21]. However, the self-consistency of the $\chi^{2}$ approach requires the residuals to be normally distributed,

$$
R_{i}=\frac{O_{i}^{\exp }-O_{i}\left(\mathbf{p}_{0}\right)}{\Delta O_{i}^{\exp }} \sim N(0,1),
$$

a condition which, even if elementary, has only recently been addressed [21] and checked in the previous analyses [18-20]. The total number of $n p$ and $p p$ data was $N=6713$. This is almost twice as in the 1993 Nijmegen analysis [11] that lacked a normality test. The normality property of the residuals has been exploited to extract the effective interaction parameters and corresponding counterterms [22] and to replicate via Monte Carlo bootstrap simulation as a means to gather more robust information on the uncertainty characteristics of fitting parameters [23]. As shown in Ref. [21] we stress that the verification of normality, Eq. (2), is essential for a meaningful propagation of the statistical error, since the uncertainty inherited from the fitted scattering data $\Delta O_{i}^{\exp }$ corresponds to a genuine statistical fluctuation. This allows us to determine the $1 \sigma$ error of the parameters $\mathbf{p}=\mathbf{p}_{0} \pm \Delta \mathbf{p}^{\text {stat }}$ and hence the 
error in the potential

$$
V_{N N}=V_{N N}\left(\mathbf{p}_{0}\right) \pm \Delta V_{N N}^{\text {stat }}
$$

which generates in turn the error in the $N N$ phase shifs $\delta=$ $\delta\left(\mathbf{p}_{\mathbf{0}}\right) \pm \Delta \delta^{\text {stat }}$ and mixing angles. Once the $N N$ potential is determined the three-body problem can be solved for the triton binding energy,

$$
\left[\sum_{i} T_{i}+\sum_{i<j} V_{N N}(i j)\right] \Psi=E_{t} \Psi
$$

where

$$
E_{t}=E_{t}\left(\mathbf{p}_{0}\right) \pm \Delta E_{t}^{\text {stat }} .
$$

Direct methods to determine $\Delta \mathbf{p}^{\text {stat }}, \Delta V_{N N}^{\text {stat }}$, and $\Delta E_{t}^{\text {stat }}$ proceed either by the standard error matrix or Monte Carlo methods (see, e.g., [24]). In Ref. [23] we have shown that the latter method is more convenient for large number of fitting parameters (typically $P=40-60$ ), and consists of generating a sufficiently large sample drawn from a multivariate normal probability distribution

$$
P\left(p_{1}, p_{2}, \ldots, p_{P}\right)=\frac{1}{\sqrt{(2 \pi)^{P} \operatorname{det} \mathcal{E}}} e^{-\frac{1}{2}\left(\mathbf{p}-\mathbf{p}_{0}\right)^{T} \mathcal{E}^{-1}\left(\mathbf{p}-\mathbf{p}_{0}\right)},
$$

where $\mathcal{E}_{i j}=\left(\partial^{2} \chi / \partial p_{i} \partial p_{j}\right)^{-1}$ is the error matrix. We generate $M$ samples $\mathbf{p}_{\alpha} \in P$ with $\alpha=1, \ldots, M$, and compute $V_{N N}\left(\mathbf{p}_{\alpha}\right)$ from which the corresponding scattering phase shifts $\delta\left(\mathbf{p}_{\alpha}\right)$ and triton binding energies $E_{t}\left(\mathbf{p}_{\alpha}\right)$ can be determined.

In our calculations we take $M=205$ samples for the smooth potential described in [21] $\left(r_{c}=3 \mathrm{fm}\right)$,

$$
V(\vec{r})=V_{\text {short }}(r) \theta\left(r_{c}-r\right)+V_{\text {long }}(r) \theta\left(r-r_{c}\right) .
$$

The long-range piece $V_{\text {long }}(\vec{r})$ contains a charge-dependent (CD) one-pion exchange (OPE) with fixed $f^{2}=0.075$ [25] and electromagnetic (EM) corrections which are kept fixed throughout the fitting process. The short-range component is

$$
V_{\text {short }}(\vec{r})=\sum_{n=1}^{21} \hat{O}_{n}\left[\sum_{i=1}^{N} V_{i, n} e^{-r^{2} /\left(2 a_{i}^{2}\right)}\right]
$$

where $\hat{O}_{n}$ are the set of operators in the extended AV18 basis [13,26-28], $V_{i, n}$ are fitting parameters, and $a_{i}=$ $a /(i+1)$ with $a=2.3035 \pm 0.0133 \mathrm{fm}$. Note that some of the $\hat{O}_{n}$ operators depend on the angular momentum, and hence contain some nonlocalities. We choose this form because the selection of the database required multiple fits, and the operator form allows a very efficient analysis and enables us to apply three-body techniques specific to this form of potential in coordinate space. For this potential $\chi^{2} / v=1.06$ and normality of residuals is verified. The potential uncertainties $\Delta V_{N N}^{\text {stat }}$ have been depicted in [21]. We have checked that statistical uncertainties in the phases and mixing angles $\Delta \delta^{\text {stat }}$ determined by the covariance matrix method (which would correspond to the limit $M \rightarrow \infty$ ) are fairly well reproduced by our $M=205$ samples when the variance of the population is used as an estimator of the error. Likewise, the uncertainties of the potential Eq. (8) obtained by the multivariate distribution, Eq. (6), are in fair agreement with our original partial wave analysis to the $3 \sigma$ self-consistent database in terms of a delta-shell potential with OPE (DS-OPE) [19] and also with the corresponding bootstrap simulation [23].

The results for $B_{t}$ for each one ot the $M=205$ Monte Carlo samples of the potential have been obtained by means of the hyperspherical adiabatic expansion method described in [29]. The angular part of the Faddeev equations is first solved for fixed values of the hyperradius $\rho$. The corresponding angular eigenfunctions $\left\{\Phi_{n}(\rho, \Omega)\right\}$ form a complete set, and it is used as a basis in order to expand the total three-body wave function $\Psi$ as

$$
\Psi=\frac{1}{\rho^{5 / 2}} \sum_{n} f_{n}(\rho) \Phi_{n}(\rho, \Omega)
$$

where $\Omega$ collects the usual five hyperangles, and where the radial wave functions $f_{n}(\rho)$ are obtained in a second step by solving a coupled set of differential radial equations where the eigenvalues of the angular part enter as effective potentials (see Ref. [29] for details).

When solving the angular part, the eigenfunctions $\Phi_{n}(\rho, \Omega)$ are expanded in terms of the hyperspherical harmonics $(\mathrm{HH})$, which contain the dependence on the quantum numbers $\left\{\ell_{x}, \ell_{y}, L, s_{x}, s_{y}, S\right\}$ of the different components included in the calculation. Obviously, $\ell_{x}$ and $s_{x}$ are the relative orbital angular momentum and spin of one of the two-body subsystems in the triton, $\ell_{y}$ is the relative orbital angular momentum between the third particle and the center of mass of the two-body system, and $s_{y}$ is the spin of the third particle. The angular momenta $\ell_{x}$ and $\ell_{y}$ couple to $L$, and $s_{x}$ and $s_{y}$ couple to the total spin $S$. Finally, $L$ and $S$ couple to the total angular momentum $1 / 2$ of the triton ground state. Together with these quantum numbers the $\mathrm{HH}$ depend of the hypermomentum $K=2 v+\ell_{x}+\ell_{y}$ $(v=0,1,2, \ldots)$.

Therefore, the convergence of the three-body wave function $\Psi$ has to be achieved at three different levels: first, in terms of the adiabatic channels included in the expansion explicitly written in Eq. (9); second, in terms of the components (with quantum numbers $\left\{\ell_{x}, \ell_{y}, L, s_{x}, s_{y}, S\right\}$ ) included in the expansion of the angular functions $\left\{\Phi_{n}\right\}$; and third, in terms of the maximum value of the hypermomentum, $K_{\max }$, used for each of the components. In the calculations presented here we have included up to 12 adiabatic terms in the expansion in Eq. (9) (typically, four or five terms are enough to get a good convergence for bound states). All the partial waves with $\ell_{x}, \ell_{y} \leqslant 5$ have been included (when increasing the number of components to $\ell_{x}, \ell_{y} \leqslant 8$ no substantial difference has been observed). Finally, three different sets of $K_{\max }$ values have been considered. We shall refer to them as sets (i), (ii), and (iii). In set (i), about $500 \mathrm{HH}$ are used in total, and $K_{\max }=50$ for the most relevant component in the three-body wave function (which corresponds to $\ell_{x}=0$ and $s_{x}=1$ between the proton and one of the neutrons, and $\ell_{y}=0$ ). In set (ii) we multiply all the $K_{\max }$-values by 2 (which means about $1000 \mathrm{HH}$ in the three-body wave function and $K_{\max }=100$ for the dominating component). Finally, in set (iii) we again multiply all the $K_{\max }$ values by 2 (therefore, about $2000 \mathrm{HH}$ in the three-body wave function and $K_{\max }=200$ for the dominating component). An appropriate choice of the $K_{\max }$ values is crucial in order to 


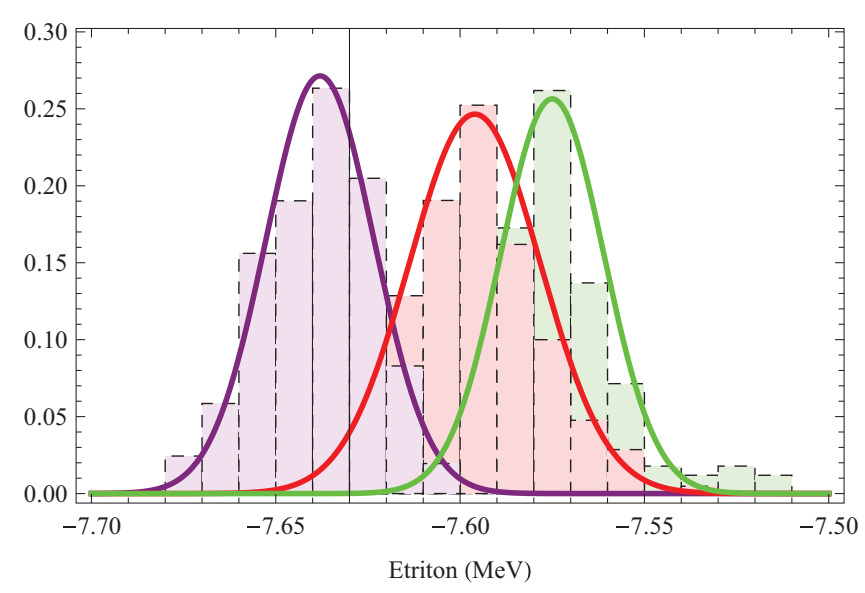

FIG. 1. (Color online) Normalized histograms representing the triton binding energy (in $\mathrm{MeV}$ ) for a sample of 205 gaussian potential parameters. From left to right the normal density probability distribution functions $N(\mu, \sigma)$ correspond to higher accuracy $E_{t}=$ $-7.638 \pm 0.0147$, intermediate accuracy $E_{t}=-7.596 \pm 0.0178$, and lower accuracy $E_{t}=-7.575 \pm 0.014$ (see main text).

optimize the computing time. An increase of the total number of $\mathrm{HH}$ in the calculation by a certain factor implies an increase of the computing time of basically the same factor squared. As an example, while a single three-body calculation with set (i) lasts for about 30 minutes, the same calculation with set (iii) requires no less than 8 hours.

The results of $B_{t}$ for the 205 Monte Carlo potential samples are summarized in the histogram of Fig. 1 for the three cases (i), (ii), and (iii) outlined above. As we see, the propagated histograms are roughly Gaussians, with quite similar widths but shifted. Thus, increasing the accuracy does not affect the error estimate substantially. For the most accurate case we get $E_{t}=-7.638(15) \mathrm{MeV}$. Taking into account the slight asymmetry in the distribution, a $\pm 1 \sigma(=68 \%)$ confidence interval can be obtained by excluding the $16 \%$ upper and lower tails. This gives the $68 \%$ range $\min E_{t} \leqslant E_{t} \leqslant \max E_{t}$ which corresponds to $\Delta E_{t}^{\text {stat }} \equiv\left(\max E_{t}-\min E_{t}\right) / 2$. There is an uncertainty in $\Delta E_{t}^{\text {stat }}$ coming from the fact that for $M=205$ we may exclude 32 or 33 values from above or below, inducing an uncertainty of $\Delta^{2} E_{t} \equiv \Delta\left(\Delta E_{t}\right)=1 \mathrm{KeV}$ on the error, so that

$$
\Delta E_{t}^{\text {stat }}=15(1) \mathrm{keV} .
$$

This is our main result, which sets a realistic precision for triton binding energy calculations and is more than two orders of magnitude larger than the experimental precision $\Delta E_{t}^{\exp }=$ $0.1 \mathrm{keV}$. The early estimate $\Delta E_{t}^{\text {th }}>40 \mathrm{keV}$ [10] was based on the ${ }^{1} S_{0}$ inverse scattering analysis using the 1980 Paris potential which has a large $\chi^{2} / v \sim 2$.

It is worth noting that the numerical error in the present calculation is $\Delta B_{t}^{\text {num }}=1 \mathrm{keV}$ which is one order of magnitude smaller than $\Delta B_{t}^{\text {stat }}=15 \mathrm{keV}$. Given that the error is dominated by the uncertainty of the input potential, we investigated if the numerical precision can be relaxed, thus reducing the computing time. Obviously, the meaning of numerical precision may depend on the method and different
TABLE I. Triton binding energy convergence in the number of channels, $N_{c}$, classified according to the orbital angular momentum of the pair $L_{\text {Pair }}$ and the spectator $l_{\text {spectator }}$ in the triton as the number of total accumulated channels, $N_{\text {Total }}$, is increased. The potential used was Monte Carlo generated. A gap indicates when the change in $E_{t}$ is smaller than the statistical uncertainty $\Delta B_{t}=15(1) \mathrm{keV}$.

\begin{tabular}{lccc}
\hline \hline$N_{c}$ & $L_{\text {Pair }} l_{\text {Spectator }}$ & $N_{\text {Total }}$ & Energy (MeV) \\
\hline 3 & Ss & 3 & Unbound \\
+2 & Sd+Ds & 5 & -7.0117 \\
+10 & Pp & 15 & -6.4377 \\
+8 & Dd & 23 & -7.4109 \\
+4 & Pf + Fp & 27 & -7.4956 \\
+10 & Ff & 37 & -7.5654 \\
+2 & Dg+Gd & 39 & -7.6178 \\
+8 & Gg & 47 & -7.6502 \\
+4 & Fh+Hf & 51 & -7.6508 \\
+10 & Hh & 61 & -7.6510 \\
\hline \hline
\end{tabular}

approaches to the three-body problem should be tried out. The convergence of the binding energy calculation in terms of partial waves (see, e.g., Ref. [5] for explicit notations) is presented in Table I for one potential taken at random and whose total energy is given by $E_{t}=-7.6510 \mathrm{MeV}$. There, an increasing number of channels is added depending on the relative orbital angular momenta $(L, l)$ of a $N N$ pair or the third spectator nucleon respectively (denoted as $\left(l_{x}, l_{y}\right)$ in the $\mathrm{HH}$ expansion above). As one can see one needs the $S s, S d, D s$ channels to get a bound triton $E_{t}=-7.0117 \mathrm{MeV}$. Within this reduced Hilbert space we get

$$
\Delta B_{t}^{\text {stat }}(S s+S d+D s)=20 \mathrm{keV} .
$$

When the $P p$ channel is added, we obtain $\Delta B_{t}^{\text {stat }}(S s+$ $S d+D s+P p)=19 \mathrm{keV}$. So, about $75 \%$ of the statistical uncertainty comes from the lowest $S s+S d+D s$ channels.

One interesting aspect from the present analysis concerns the statistical correlation analysis of the $N N$ gaussian potential parameters, as this helps to pin down what does fix the current precision. We find that correlations are never larger than 0.4 , but since the Gaussian potential parameters themselves are strongly correlated, there is still the possibility that more global parameters such as volume integrals or low energy scattering parameters would show a clearer pattern.

The precision has been a recurrent topic within the present context, and much of the effort was originally directed with the purpose of establishing the need of $3 N$ forces within the numerical precision of the calculations. For instance, one needs 34 channels up to angular momentum $J_{\text {pair }} \leqslant 4$ to obtain $\Delta E_{t}^{\text {num }}=10 \mathrm{keV}$ [7]. Within this numerical precision the triton binding energy obtained by Faddeev calculations has been found to be $8.00,7.62,7.63,7.62,7.72 \mathrm{MeV}$ for the $\mathrm{CD}$ Bonn [30], Nijm-II, Reid93, Nijm-I, and AV18 [31] potentials respectively. The covariant spectator model has produced the closest binding energy $8.50 \mathrm{MeV}$ to experiment precisely when the $N N \chi^{2}$ becomes smallest. The spread of values in $B_{t}$, allowed by the theorem of Glöckle and Polyzou [32], is coming from off-shell ambiguities. The theorem, however, does not predict quantitatively the dispersion, which yields 
$B_{t}=7.85(34) \mathrm{MeV}$ [experimentally $B_{t}=8.4820(1) \mathrm{MeV}$ ]. The similarity of the databases but the different potential forms suggests calling this a systematic error, i.e., $\Delta B_{3}^{\text {syst }}=340 \mathrm{keV}$. In previous estimates a value of $B_{t}=7.62(1)$ was obtained using the NijmII, AV18, and Reid93 local potentials fitted to the same database [31]. This was extrapolated to be $B_{t}=$ 7.6(1) [33] from an inverse scattering analysis of Nijmegen phases up to $T_{\mathrm{LAB}}=300 \mathrm{MeV}$ based on a local potential, the error stemming from the high energy extrapolation. We note that these are essentially systematic error estimates.

It is worth addressing what would be the uncertainties in the statistical error estimate due to a different choice of the $N N$ potential. Actually, we expect this to be of the order of relative $\Delta B_{t}^{\text {syst }} / B_{t} \sim 340 \mathrm{keV} / 8482 \mathrm{keV} \sim 0.04$ which turns out to be about a half than our relative uncertainty on the error estimate, Eq. (10), namely $\Delta^{2} B_{t} / \Delta B_{t}=1 \mathrm{keV} / 15 \mathrm{keV} \sim 0.07$. Thus, the uncertainty due to the particular form of the $N N$ potential, be it local or nonlocal, is not expected to modify our main result.

A high precision calculation with the AV18 potential using the $\mathrm{HH}$ expansion method was carried out by the Pisa group [9] leading to the sequence of values $B_{t}=7.59267,7.61227,7.61786,7.61809,7.61812 \mathrm{MeV}$ for $N_{c}=8,14,18,22,26$ channels respectively. According to our error estimate of $\Delta B_{t}=0.02 \mathrm{MeV}$ one could stop already at $N_{c}=8$ for a realistic precision. Similar remarks apply to [8] where $\Delta B_{t}^{\text {num }}=0.1 \mathrm{keV}$. Based on general arguments, attempts have also been made to quantify the systematic uncertainties in nuclear bindings stemming from $N N$ scattering $[16,26,27])$ yielding $\Delta E^{\text {sys }} / A=100-500 \mathrm{keV}$, in rough agreement with the more sophisticated three-body estimate of $340 \mathrm{KeV}$ above. This suggests using the present calculation as a benchmark in approximate error estimates, sidestepping the full-fledged calculation.
From a more general perspective, there is an ongoing effort to quantify the uncertainties in nuclear physics [34,35] as a means to establish the real predictive power of the theory. While this topic is presently in its infancy, from a theoretical point of view and the inferred predictive power, errors in ab initio calculations can be grouped into three main categories: (i) the input information (in our case the $N N$ scattering experimental data), (ii) the method of solution and its numerical precision, and (iii) the form (e.g., local or nonlocal) of the interaction in the unknown region. We have denoted these errors as $\Delta E^{\text {stat }}, \Delta E^{\text {num }}$, and $\Delta E^{\text {syst }}$ respectively. Assuming that these sources of error are independent of each other, we expect the total theoretical uncertainty to be given by

$$
\left(\Delta E^{\text {th }}\right)^{2}=\left(\Delta E^{\text {stat }}\right)^{2}+\left(\Delta E^{\text {num }}\right)^{2}+\left(\Delta E^{\text {syst }}\right)^{2} .
$$

Clearly, the total error is dominated by the largest one. So, it makes sense either to reduce the largest source of uncertainty or to tune all uncertainties to a similar level. This sets a realistic limit of predictive power in ab initio calculations, which we find to be $\Delta E_{t}^{\text {th }} \geqslant 15(1) \mathrm{keV}$. While the use of realistic potentials has been a must in few-body calculations, we note that the physical precision of the calculation is finite and will definitely have sizable consequences in large scale calculations in nuclear physics. Given the large systematic uncertainties, the theoretical calculation of the triton binding energy provides a good example of a precise but inaccurate quantity.

We thank Andreas Nogga for discussions and communications and for drawing our attention to Ref. [10]. This work is supported by Spanish Ministerio de Economía y Competitividad (Grants No. FIS2011-23565 and No. FIS201124149) and Junta de Andalucía (Grant No. FQM225). R.N.P. is supported by a Mexican CONACYT grant.
[1] H. Bethe and R. Bacher, Rev. Mod. Phys. 8, 82 (1936).

[2] J. Blatt and V. Weisskopf, Theoretical Nuclear Physics (John Wiley and Sons, New York, 1952).

[3] R. Amado, Annu. Rev. Nucl. Sci. 19, 61 (1969).

[4] L. M. Delves and A. Phillips, Rev. Mod. Phys. 41, 497 (1969).

[5] Y. Wu, S. Ishikawa, and T. Sasakawa, Few-Body Syst. 15, 145 (1993).

[6] W. Glöckle, The Quantum Mechanical Few-Body Problem (Springer, Berlin, 1983).

[7] C. R. Chen, G. L Payne, J. L. Friar, and B. F. Gibson, Phys. Rev. C 31, 2266 (1985).

[8] C. Elster, W. Schadow, A. Nogga, and W. Gloeckle, Few Body Syst. 27, 83 (1999).

[9] A. Kievsky, L. Marcucci, S. Rosati, and M. Viviani, Few-Body Syst. 22, 1 (1997).

[10] R. Adam, H. Fiedeldey, S. Sofianos, and H. Leeb, Nucl. Phys. A 559, 157 (1993).

[11] V. G. J. Stoks, R. A. M. Kompl, M. C. M. Rentmeester, and J. J. de Swart, Phys. Rev. C 48, 792 (1993).

[12] V. G. J. Stoks, R. A. M. Klomp, C. P. F. Terheggen, and J. J. de Swart, Phys. Rev. C 49, 2950 (1994).
[13] R. B. Wiringa, V. G. J. Stoks, and R. Schiavilla, Phys. Rev. C 51, 38 (1995).

[14] R. Machleidt, Phys. Rev. C 63, 024001 (2001).

[15] F. Gross and A. Stadler, Phys. Rev. C 78, 014005 (2008).

[16] R. Navarro Pérez, J. E. Amaro, and E. Ruiz Arriola, Phys. Lett. B 724, 138 (2013).

[17] A. Ekström, G. Baardsen, C. Forssén, G. Hagen, M. Hjorth-Jensen et al., Phys. Rev. Lett. 110, 192502 (2013).

[18] R. Navarro Pérez, J. E. Amaro, and E. Ruiz Arriola, Phys. Rev. C 88, 024002 (2013).

[19] R. Navarro Pérez, J. E. Amaro, and E. Ruiz Arriola, Phys. Rev. C 88, 064002 (2013).

[20] R. Navarro Pérez, J. E. Amaro, and E. Ruiz Arriola, Phys. Rev. C 89, 024004 (2014).

[21] R. Navarro Pérez, J. E. Amaro, and E. Ruiz Arriola, Phys. Rev. C 89, 064006 (2014).

[22] R. Navarro Pérez, J. E. Amaro, and E. Ruiz Arriola, arXiv:1406.0625 [J. Phys. G (to be published)].

[23] R. Navarro Pérez, J. E. Amaro, and E. Ruiz Arriola, arXiv:1407.3937 [Phys. Lett. B (to be published)]. 
[24] J. Nieves and E. Ruiz Arriola, Eur. Phys. J. A 8, 377 (2000).

[25] V. Stoks, R. Timmermans, and J. J. de Swart, Phys. Rev. C 47, 512 (1993).

[26] R. Navarro Pérez, J. E. Amaro, and E. Ruiz Arriola, arXiv:1202.6624.

[27] R. Navarro Pérez, J. E. Amaro, and E. Ruiz Arriola, PoS(QNP 2012)145.

[28] J. E. Amaro, R. Navarro Pérez, and E. Ruiz Arriola, Few Body Syst. 55, 977 (2014).

[29] E. Nielsen, D. Fedorov, A. Jensen, and E. Garrido, Phys. Rep. 347, 373 (2001).
[30] R. Machleidt, F. Sammarruca, and Y. Song, Phys. Rev. C 53, R1483(R) (1996).

[31] J. L. Friar, G. L. Payne, V. G. J. Stoks, and J. J. de Swart, Phys. Lett. B 311, 4 (1993).

[32] W. N. Polyzou and W. Glöckle, Few-Body Syst. 9, 97 (1990).

[33] B. F. Gibson, H. Kohlhoff, H. von Geramb, and G. Payne, Phys. Rev. C 51, R465(R) (1995).

[34] J. Dudek, B. Szpak, B. Fornal, and A. Dromard, Phys. Scr. 2013, 014002 (2013).

[35] J. Dobaczewski, W. Nazarewicz, and P.-G. Reinhard, J. Phys. G 41, 074001 (2014). 